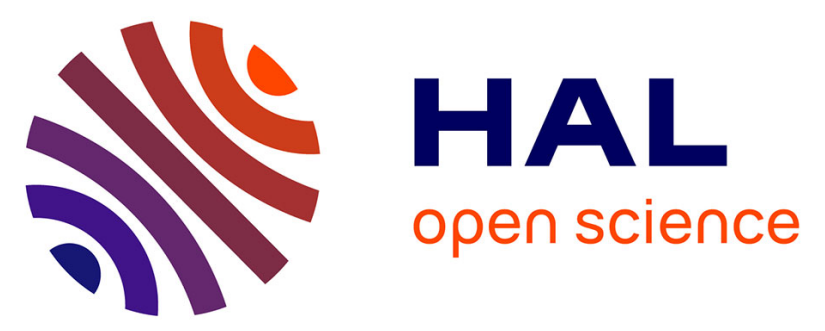

\title{
Influence of the Number of Predicted Words on Text Input Speed in Participants With Cervical Spinal Cord Injury
}

Samuel Pouplin, Nicolas Roche, Isabelle Vaugier, Antoine Jacob, Marjorie Figere, Sandra Pottier, Jean-Yves Antoine, Djamel Bensmail

\section{To cite this version:}

Samuel Pouplin, Nicolas Roche, Isabelle Vaugier, Antoine Jacob, Marjorie Figere, et al.. Influence of the Number of Predicted Words on Text Input Speed in Participants With Cervical Spinal Cord Injury: Influence of the number of predicted words in persons with SCI. Archives of Physical Medicine and Rehabilitation, 2015, 97 (259-265), 10.1016/j.apmr.2015.10.080 . hal-01246707

\section{HAL Id: hal-01246707 https://hal.science/hal-01246707}

Submitted on 19 Dec 2015

HAL is a multi-disciplinary open access archive for the deposit and dissemination of scientific research documents, whether they are published or not. The documents may come from teaching and research institutions in France or abroad, or from public or private research centers.
L'archive ouverte pluridisciplinaire $\mathbf{H A L}$, est destinée au dépôt et à la diffusion de documents scientifiques de niveau recherche, publiés ou non, émanant des établissements d'enseignement et de recherche français ou étrangers, des laboratoires publics ou privés. 
1 TITLE: Influence of the number of predicted words on text input speed in

2 participants with cervical spinal cord injury.

3

4 SHORT TITLE: Influence of the number of predicted words in persons with

5 SCI

6

7 AUTHORS

8 Samuel POUPLIN, OT, PhD Student ${ }^{1-4}$

9 Nicolas ROCHE, $\mathrm{MD}, \mathrm{PhD}^{3-5}$

10 Isabelle VAUGIER, Biostatistician ${ }^{4}$

11 Antoine JACOB, OT, $\mathrm{MSc}^{1}$

12 Marjorie FIGERE, $\mathrm{ARC}^{4}$

13 Sandra POTTIER, Project Manager ${ }^{4}$

14 Jean-Yves ANTOINE ${ }^{6}$,

15 Djamel BENSMAIL, MD, $\mathrm{PhD}^{1-4}$

16

$17{ }^{1}$ New Technologies Plate-Form, AP-HP, Raymond Poincaré Teaching Hospital, 18 Garches, France.

$19{ }^{2}$ Physical Medicine and Rehabilitation Department, AP-HP, Raymond Poincaré

20 Teaching Hospital, Garches, France. 
$21{ }^{3}$ Inserm Unit 1179, Team 3: Technologies and Innovative Therapies Applied to

22 Neuromuscular diseases, University of Versailles St- Quentin-en-Yvelines,

23 France.

$24{ }^{4}$ Clinical Innovations Center 1429, AP-HP, Raymond Poincaré Teaching Hospital,

25 Garches, France.

$26{ }^{5}$ Physiology-Functional Testing Ward, AP-HP, Raymond Poincaré Teaching

27 Hospital, Garches, France

$28{ }^{6}$ University François Rabelais of Tours, Tours, France, LI Lab

29 


\section{$30 \quad$ ABSTRACT}

31 Objective

32 To determine if the number of words displayed in the Word Prediction Software

33 (WPS) list affects Text Input Speed (TIS) in people with cervical Spinal Cord

34 Injury (SCI) and if any influence is dependent on the level of the lesion.

35 Design

36 A cross-sectional trial.

$37 \quad$ Setting

38 A rehabilitation center in France.

39 Participants

40 Ninety persons with cervical SCI fulfilled the inclusion/exclusion criteria, 45 of

41 whom agreed to participate. Lesion level was high (C4 and C5 Asia A or B) for

4215 participants (high lesion group) and was between C6 and C8 Asia A or B for

4330 participants (low lesion group).

$44 \quad \underline{\text { Methods }}$

45 TIS was evaluated during 4. 10-minute copying tasks:

46 -without WPS (Without)

47 -with a display of 3 predicted words (3Words)

48 -with a display of 6 predicted words (6Words)

49 -with a display of 8 predicted (8Words) 


\section{Outcome Measures}

51 During the 4 copying tasks, TIS was measured objectively (characters per minute,

52 number of errors) and subjectively through subject report (fatigue, perception of

53 speed, cognitive load, satisfaction)

\section{$54 \quad$ Results}

55 For participants with low cervical SCI, text input speed without WPS was faster

56 than with WPS, regardless of the number of words displayed $(\mathrm{p}<0.001)$. For

57 participants with high cervical SCI, the use of WPS did not influence TIS

$58(\mathrm{p}=0.99)$. There was no influence of the number of words displayed in a word

59 prediction list on TIS, however perception of TIS differed according to lesion

60 level.

61 Conclusion

62 For persons with low cervical SCI, a small number of words should be displayed,

63 or WPS should not be used at all. For persons with high cervical SCI, a larger

64 number of words displayed increases the comfort of use of WPS.

$65 \underline{\text { Key words }}$

66 Cervical spinal cord injury, text input speed, word prediction software, words

67 displayed 
69 ABREVIATIONS:

70 SCI: Spinal Cord Injury

71 TIS: Text Input Speed

72 WPS: Word Prediction Software

73 CPM: Characters per minute

74 SD: Standard Deviation

75

76

77

78 


\section{Introduction}

80 The use of technology is essential for the social and professional integration of

81 persons with cervical spinal cord injury (SCI) ${ }^{1}$. Likewise, the emergence of new

82 interfaces such as tablets and smartphones have changed how people

83 communicate and use the Internet ${ }^{2}$. However, access to Internet and social

84 websites, which is mainly based on text input, can be difficult, especially for

85 persons with high cervical SCI. A variety of devices (infrared cameras, onscreen

86 keyboards etc.) have been designed to facilitate computer use, depending on the

87 level of the lesion ${ }^{34.5 .6 .7 .8 .9}$. Despite the use of these devices, text input remains

88 laborious with a mean text input speed (TIS) of 5 words per minute ${ }^{10}$ compared

89 with $15-20$ words per minute in able-bodied people ${ }^{10}$. Several methods have

90 been developed to increase TIS ${ }^{11} 9121314.15$, such as speech recognition systems ${ }^{16}$

91 or word prediction software (WPS). These methods are recommended by health-

92 related professionals ${ }^{17}$ to increase TIS. However, in a noisy home environment,

93 the use of a speech recognition system may be compromised. Also, some people

94 want to keep their privacy when they dictate a text. Thus WPS may be a solution

95 to compensate for some of the disadvantages of speech recognition software. WPS

96 display a list of predicted words that correspond to the word currently being typed

97 by the user. If one of the predictions is correct, the user selects the corresponding

98 word in the list, thereby avoiding typing each letter of the word (keystroke

99 saving $^{18}$ ). WPS can be customized, for example by changing the number of words

100 displayed. 
102 Data in the literature are conflicting regarding the influence of WPS on TIS, with 103 some studies showing decreases of up to $71 \%$ and others showing increases of up

104 to $45 \% 19202122232425$. The main reason suggested for these differences is the

105 increase in cognitive load caused by the visual search for words in the prediction

106 list. This suggests that the number of words in the prediction list affects TIS.

107 A study in healthy people showed that keystroke savings are significantly related

108 to the number of words displayed ${ }^{26}$. However, since selection time increases with

109 the number of words in the list, the benefits provided by keystroke savings may be

110 cancelled out. A simulation study showed that each additional word displayed in

111 the prediction list increases search time by 150 milliseconds ${ }^{27}$. Moreover, there is

112 only a slight increase in keystroke savings between 6 and 11 words. According to

113 these studies, the best compromise between keystroke savings and cognitive load

114 appears to be 5 or 6 words ${ }^{27}$.

115 A preliminary study ${ }^{17}$ carried out in our group showed that health-related

116 professionals most frequently set 6 words for their patients, similarly to data in the

117 literature. However, an unpublished study in our department showed that persons

118 with cervical SCI tended to set a display of 8 words for themselves.

120 These results suggest that the number of words displayed in the predicted list is

121 important, however, the optimal number has not yet been determined in a large

122 sample of persons with cervical SCI. 
123 The aims of this study were therefore to determine if the number of words in the

124 predicted list influences TIS in a large population of persons with cervical SCI

125 Asia A or B and if this number was influenced by the level of cervical lesion.

126 Based on data in the current literature, we hypothesized that 6 words would be

127 optimal.

128

129 Method

$130 \quad \underline{\text { Participants }}$

131 This prospective cross-sectional study was carried out between October

1322013 and March 2014. Persons with cervical SCI followed up in the department of

133 physical medicine and rehabilitation of a Teaching Hospital were included by a

134 physician and an occupational therapist if they were over 18 years old, had a SCI

135 between $\mathrm{C} 4$ and $\mathrm{C} 8$ Asia A or B, were computer users, could read and write

136 French and were not regular user of WPS. They were excluded if they had

137 cognitive, linguistic or visual impairments. The study was approved by the local

138 ethics committee (CPP Ile-de-France, Saint Germain en Laye) and all subjects

139 provided written informed consent before participation. Data collection was

140 performed by an occupational therapist and took place in the department of

141 physical medicine and rehabilitation in the teaching hospital in which the patient

142 was recruited.

143 Participants were included in one of two distinct groups, depending on their

144 lesion level: 
146 -A low lesion group for persons with C6, C7 or C8 Asia A or B tetraplegia.

147 The distinction between the high lesion group and the low lesion group was

148 made because persons with lesions at or below C6 have sufficient wrist extension

149 to use a standard keyboard ${ }^{28}$.

$151 \quad \underline{\text { Materials }}$

152 To standardize the evaluation conditions, a Dell-XPS computer, equipped with a

153 KeyVit Onscreen Keyboard and Skippy WPS were used. Skippy was chosen as it

154 has been shown to be the WPS which is the most prescribed and used ${ }^{17}$.

155 Participants who used an onscreen keyboard used their usual pointing devices

156 (head-controlled).

157 The WPS was configured to display the list of words horizontally at the top of the

158 screen, as is most frequent. The number of words (3, 6 and 8) was chosen based

159 on results from our previous study on the use of WPS and data in the literature.

160 Two parameters were not activated: automatic learning of new words and a faster

161 presentation of the words most frequently used (frequency of use). It has been

162 shown that most persons with cervical SCI use commercial WPS without such

163 advanced settings ${ }^{17}$. Words were thus displayed alphabetically in the prediction

164 list, as is the case in the majority of WPS.

166 Procedures. 
167 Firstly, the use of WPS was explained to each participant. Then, each participant

168 was allowed a 5 minute-training period using the WPS in a copying task. Finally,

169 each participant underwent a single evaluation session involving 4 copying task

170 conditions. The conditions were randomly assigned to avoid bias associated with

171 fatigue:

172 -without WPS (Without)

173 -with 3 predicted words (3Words)

$174 \quad$-with 6 predicted words (6Words)

175 -with 8 predicted (8Words)

176 The randomization was performed using dedicated software and a system of

177 sealed envelopes was used for allocation. A maximum of 10 minutes was allowed

178 for each task and participants were given a five-minute break between each task.

179 Four 500-word texts of similar complexity were used, drawn from a speech and

180 language therapy book $^{29}$. The average word length was $5.1 \pm 0.5(\mathrm{SD})$.

181 The length of each text was deliberately too long for it to be copied in 10 minutes.

182 The evaluation was therefore stopped after 10 minutes. The texts were randomly

183 allocated in order to ensure that the same text was not associated with the same

184 copying task.

185 Participants were instructed to use the WPS but no instructions regarding

186 strategies of use were given. Errors could be corrected.

187 All assessments were videotaped and the videos were used for the analysis. All

188 the evaluations were performed by the same investigator to limit bias. 
190 Outcome Measures.

191 During the 4 copying tasks, TIS was calculated as follows:

192 Objective evaluations

193 Characters per minute (cpm): Number of characters typed in ten minutes

194 divided by 10, including punctuation marks, spaces, backspace, selection errors,

195 and correction times.

196 Item selection speed (item per minute): Number of items selected in ten minutes

197 divided by 10 including punctuation marks, spaces, backspaces, arrow keys and

198 keys used to select words in the word prediction list.

199 Number of errors and rate of word prediction use: The number of errors and

200 number of predicted words selected from the word prediction list in ten minutes

201 were calculated from the videos.

202 Subjective evaluations (self-evaluations).

203 Fatigue was evaluated using a 0-10 point visual analog scale (VAS) before and

204 after every task (0: no fatigue - 10: exhaustion)

205 Perception of speed and cognitive load were evaluated using a 0-10 point VAS.

206 For perception of speed, 0: very slow - 10: very fast; for cognitive load, 0: low

207 cognitive load - 10: high cognitive load.

208 Satisfaction was evaluated using a 0-5 point VAS (0: not satisfied/5: very

209 satisfied)

210 
211 Data Analysis

212 Descriptive statistics (mean \pm standard deviation) were used to describe continuous

213 variables and frequencies for categorical variables.

214 A Wilcoxon test was used to analyze differences in age and education level

215 between the low and high lesion groups.

216 A Chi square test was used to analyze differences in gender, frequency of use of

217 word processing and frequency of computer use between the low and high lesion

218 groups.

219 The objective and subjective data relating to TIS followed a normal distribution

220 (Shapiro-Wilk-test) and thus parametric tests were used. To compare the

221 influence of the number of words displayed in the prediction list on TIS, item

222 selection speed, number of errors, rate of word prediction use, satisfaction,

223 cognitive load and perception of speed, a repeated-measures ANOVA with two

224 factors: type of assessment (Without/3Words/6Words/8Words) and lesion level

225 (high/low) was used. A post-hoc Fisher's least significant difference (LSD) test

226 was carried out on significant results. For the analysis of the high lesion group, we

227 used a repeated-measures ANOVA with two factors: type of assessment

228 (Without/3Words/6Words/8Words) and devices used (standard

229 keyboard/onscreen keyboard + Trackball/ onscreen keyboard + Infrared camera).

230 The level of significance was fixed at $\mathrm{p}<0.05$. Data were analyzed using

231 STATISTICA 10 software-StatSoft. Inc software (Tulsa, USA). 


\section{Results}

\section{Demographic results}

235 Ninety persons with cervical SCI fulfilled the inclusion/exclusion criteria, of

236 whom 45 agreed to participate in this study (35 males and 10 females; mean age

23739.6 (SD10) years). Mean time since lesion of the overall group was 10.6 (SD8)

238 years.

239 Fifteen participants were included in the high lesion group (14 males and 1

240 female, mean age 40.9 (SD9) years). Ten participants had used a computer for

241 over 10 years, 2 between 5 and 10 years, 2 between 1 and 5 years and 1 for less

242 than 1 year. Six subjects used infrared tracking technology and 9 used a trackball

243 controlled by the chin. All used onscreen keyboards. Thirteen subjects used word

244 processing programs regularly ( $>3$ times/week) and 2 did not ( $\leq 3$ times/month).

245 Thirty participants were included in the low lesion group (21 males and 9

246 females, mean age 39.5 (SD11) years. Twenty-six participants had used a

247 computer for over 10 years, 3 participants between 5 and 10 years and 1 between

$248 \quad 1$ and 5 years. All participants used a standard keyboard without splints and used

249 word processing programs regularly (>3 times/week).

250

251 There were no significant differences between groups for age, gender, years of

252 education and frequency of use of word processing programs. However,

253 participants in the low lesion group used the computer more frequently than

254 participants in the high lesion group $(\mathrm{p}<0.001)$. 


\section{Results of objective evaluations}

257 TIS (Characters per minute)

258 -Insert table 1

259 There was a significant effect of condition on TIS

260 (Without/3Words/6Words/8Words $)(\mathrm{F}(3,129)=8.64 ; \mathrm{p}<0.001)$; there was also a

261 significant effect of lesion level $(\mathrm{F}(1,43)=27.6 ; \mathrm{p}<0.001)$ and a significant

262 interaction between the 2 factors $(\mathrm{F}(3,129)=8.89, \mathrm{p}<0.001)$.

263 The post-Hoc analysis indicated that participants with low lesions inputted text

264 faster than participants with high lesions. For participants with low lesions, text

265 input was faster without WPS than with WPS (3Words/6Words/8Words)

266 regardless of the number of words displayed $(\mathrm{p}<0.001)$. For participants with high

267 lesions, there was no influence of WPS (3Words/6Words/8Words) on TIS

$268(\mathrm{p}=0.99)$.

269 In the high lesion group, there was no significant effect of condition on TIS

$270(\mathrm{~F}(3,39)=0.2 ; \mathrm{p}=0.89)$; however, there was a significant effect of the device used

$271(\mathrm{~F}(1,13)=11,2 ; \mathrm{p}=0.005$ with no interaction between the 2 factors $(\mathrm{F}(3,39)=0.75$;

$272 \mathrm{p}=0.52)$

273

$274 \quad$ Number of Errors

275 -Insert table 2 
276 There was a significant effect of lesion level on the number of errors

$277(\mathrm{~F}(1,43)=35.3 ; \mathrm{p}<0.001)$. However, there was no influence of condition

$278(\mathrm{~F}(3 ; 129)=0.9 ; \mathrm{p}=0.43)$ and no interaction between the 2 factors

$279 \quad(\mathrm{~F}(3,129)=0.18, \mathrm{p}=0.90)$.

280 The Post-Hoc analysis indicated that the high lesion group made fewer errors than

281 the low lesion group $(\mathrm{p}<0.001)$. There was no influence of condition $(\mathrm{p}=0.44)$ on

282 the number of errors in either group.

283 In the high lesion group, there was no significant effect of condition on the

284 number of errors $(F(3,39)=1.5 ; p=0.22)$, no significant effect of the device used

$285(\mathrm{~F}(1,13)=0.002 ; \mathrm{p}=0.96)$ and no interaction between the 2 factors $(\mathrm{F}(3,39)=1.6$;

$286 \mathrm{p}=0.20)$

287

288

289 Item selection speed.

290 -Insert table 3

291 There was a significant effect of condition on item selection speed

292 (Without/3Words/6Words/8Words)(F(3,129)=7.84; $<<0.001)$. There was also a

293 significant effect of lesion level $(\mathrm{F}(1,43)=28.76 ; \mathrm{p}<0.001)$ and a significant

294 interaction between the 2 factors $(\mathrm{F}(3,129)=11.11 ; \mathrm{p}<0.001)$.

295 The Post-Hoc analysis indicated that participants with high lesions had a higher

296 key selection speed than participants with low lesions. Key selection speed was 
297 higher without WPS for participants with low lesions $(\mathrm{p}<0.001)$ whereas there

298 were no differences between conditions for the high lesion group $(p=0.99)$.

299 In the high lesion group, there was no significant effect of condition on item

300 selection speed $(\mathrm{F}(3,39)=0.9 ; \mathrm{p}=0.44)$. However, there was a significant effect of

301 the device used $(\mathrm{F}(1,13)=9.8 ; \mathrm{p}=0.007)$ with no interaction between the 2 factors

$302(\mathrm{~F}(3,39)=0.8 ; \mathrm{p}=0.49)$

303

$304 \quad$ Rate of word prediction use.

305 -Insert table 4

306 There was a significant effect of lesion level on rate of use of word prediction.

$307(\mathrm{~F}(1,43)=5.6 ; \mathrm{p}=0.02)$. There was no influence of condition $(\mathrm{F}(2,86)=1.6 ; \mathrm{p}=0.18)$

308 and no interaction between condition and lesion level $(\mathrm{F}(2,86)=2.6, \mathrm{p}=0.07)$.

309 The Post Hoc analysis showed no interaction between low and high lesions

$310(\mathrm{p}=0.33)$ or between lesion level and condition $(\mathrm{p}=0.99)$.

311 In the high lesion group, there was no significant effect of condition on rate of use

312 of word prediction $(\mathrm{F}(2,26)=1.49 ; \mathrm{p}=0.24)$; However, there was a significant

313 effect of the device used $(\mathrm{F}(1,13)=5.6 ; \mathrm{p}=0.003$ with no interaction between the 2

314 factors $(\mathrm{F}(2,26)=2.65 ; \mathrm{p}=0.09)$

316 Results of the subjective evaluations

317 Fatigue 
318 There was no significant effect of condition $(\mathrm{F}(3,129)=1.86) ; \mathrm{p}=0.97)$ or lesion

319 level $(\mathrm{F}(1,43)=0.2 ; \mathrm{p}=0.65)$ and no interaction between the 2 factors

$320 \quad(\mathrm{~F}(3,129)=1.86 ; \mathrm{p}=0.13)$.

$321 \quad$ Perception of TIS

322 There was a significant effect of condition $(F(2,86)=4.91 ; \mathrm{p}<0.001)$ and lesion

323 level $(\mathrm{F}(1,43)=6.82 ; \mathrm{p}=0.01)$ with no interaction between the 2 factors

$324(\mathrm{~F}(2,86)=2.34 ; \mathrm{p}=0.10)$

325 The Post-Hoc analysis indicated that, for the low lesion group, participants

326 perceived text input as faster with a display of 3 words compared to 8 words

$327(\mathrm{p}=0.003)$. Participants with high lesions perceived text input as faster with a

328 display of 6 and 8 words than participants with low lesions (respectively

$329 \mathrm{p}=0.03 ; \mathrm{p}<0.001)$.

$330 \quad$ Cognitive load

331 There was no influence of condition $(\mathrm{F}(2,86)=1.42 ; \mathrm{p}=0.24)$ or lesion level

$332(\mathrm{~F}(1,43)=0.91 ; \mathrm{p}=0.35)$ and no interaction between the 2 factors

$333(\mathrm{~F}(2,86)=1.33 ; \mathrm{p}=0.26)$.

334 Satisfaction

335 There was no influence of condition $(\mathrm{F}(2,86)=0.31 ; \mathrm{p}=0.73)$. There was a

336 significant effect of lesion level $(\mathrm{F}(1,43)=5.97 ; \mathrm{p}=0.02)$ and a significant effect

337 between the 2 factors $(\mathrm{F}(2,86)=3.25 ; \mathrm{p}=0.04)$. The Post-Hoc analysis indicated that

338 for the high lesion group, satisfaction with 8 Words was higher than for the low

339 lesion group $(\mathrm{p}=0.01)$ 


\section{Discussion}

342 We found in this study that the influence of WPS on text input speed depended on

343 the lesion level of the user. TIS was faster without WPS for participants with low

344 lesions, whatever the number of words displayed, while there was no influence of

345 WPS in participants with high lesions. These results refute our hypothesis and

346 contrast with previous results in the literature.

348 Influence of WPS on TIS.

349 The influence of WPS on TIS differed depending on the level of cervical SCI.

350 This result was further confirmed by the rate of word prediction use in each

351 group.

\section{In each group.}

353 For the low lesion group, the decrease in TIS with WPS was associated with a

354 decrease in key selection speed, even if the cognitive load was not higher with

355 WPS in this group. However, this is in accordance with previous studies ${ }^{19.22}$ and

356 could relate to the necessity to search for predicted words on the computer screen

357 while using a physical keyboard.

358 For the high lesion group, TIS, item selection speed and cognitive load were not

359 affected by WPS, whatever the device used. These results therefore suggest that

360 not only is the use of WPS not effective to increase TIS in people with cervical

361 SCI, it may actually have a negative influence on TIS. However, the adjustment of 
362 other settings could change the influence of WPS on TIS. In another study

363 conducted by our team (in press), we showed that the activation of "frequency of

364 use" increased TIS in persons with high cervical SCI. The difference in results

365 between the two groups may relate to the fact that the cognitive load induced by

366 the visual search for words in the prediction list is lower with the use of an

367 onscreen keyboard since a smaller degree of visuospatial exploration is required

368 than for a standard keyboard (used by the low SCI level group). Tam et al (2009)

369 confirmed this hypothesis since they found that people with cervical SCI who

370 used an external device to display the word prediction list near the standard

371 keyboard had to look at their fingers when they typed ${ }^{30}$.

\section{Between group comparison}

373 There were fewer text input errors in the high lesion group than the low lesion

374 group. This could be the result of the lower TIS of the high lesion group along

375 with the fact that use of an onscreen keyboard requires a smaller degree of

376 visuospatial exploration.

377 The lack of influence of WPS on fatigue in both groups contradicts data in the

378 literature. WPS has previously been shown to reduce fatigue in persons with

379 cerebral palsy ${ }^{31}$. This difference might be related to the fact that persons with

380 cervical SCI have lower levels of fatigue than persons with brain injury. This

381 should, however be evaluated in further comparative studies. The results of the

382 present study may also have been affected by the fact that the "frequency of use"

383 and "learning new words" parameters were disabled. This could affect TIS, 
384 fatigue and the number of errors. It would therefore be interesting to study the

385 influence of these parameters more specifically in future studies.

387 Influence of the number of words displayed on TIS.

388 We initially hypothesized that the number of words displayed in the prediction list

389 would influence TIS. However, there was no influence of the number of words

390 displayed on TIS or on key selection speed in either group, whatever the device

391 used. Similarly, there was no influence on rate of word prediction use. These

392 results contrast with previous results in the literature. Koester found that a display

393 of 5 or 6 words is the best compromise between increasing TIS and cognitive

$394 \operatorname{load}^{27}$. This difference may be related to differences in methodology and the fact

395 that the sample of participants with cervical SCI was larger in the present study.

396 Participants with low lesions perceived text input to be faster with a display of 3

397 words rather than 8 words. This may be related to the fact that a shorter list

398 requires a shorter visual search time. In contrast, satisfaction was higher with a

399 display of 8 words for participants with high lesions. The higher TIS of

400 participants with low cervical SCI may make reducing visual search time a

401 priority while the use of a virtual keyboard by participants with high lesions and

402 the low associated TIS may induce a preference for a greater choice of words and

403 greater key stroke savings. However, it must be noted that altering the number of

404 words displayed only affected the perception of TIS but had no objective

405 influence. 


\section{Study limitations}

409 The difference in the number of subjects and the difference in the frequency of

410 computer use in the high and low cervical SCI groups could constitute a bias in

411 the interpretation of the results. However, any such bias appears to have had a

412 minimal impact since the variability of the two groups was almost similar. No

413 studies found in the literature have evaluated the influence of lesion level on TIS.

414 This study on word prediction software involved the largest sample of persons

415 with cervical SCI currently available in the literature and thus the results are

416 worthy of note.

417 Moreover, the use of different computer access devices in the high lesion group

418 influenced text input speed and item selection speed. Nevertheless, the results

419 suggest that the impact of these different devices on the influence of word

420 prediction software and the number of words displayed was small. We found no

421 influence of the number of words displayed on TIS in the high lesion group, and

422 no influence of the WPS on TIS as a function of the type of device used. In

423 addition, the lack of validation of the visual analogue scales used may constitute a

424 limitation for the analysis and the interpretation of results.

425 The alteration of other parameters such as the frequency of words displayed may

426 influence TIS by increasing the relevance of the displayed words. Moreover, lack 
427 of training in the use of WPS could also influence TIS. The influence of training

428 should be considered in future studies.

\section{Conclusions}

431 The influence of the number of words displayed in a word prediction list on TIS

432 differed depending on the level of cervical SCI. The use of WPS decreased TIS in

433 participants with low lesions, whatever the number of words displayed. In

434 participants with high lesions, there was no influence of WPS on TIS and no

435 influence of the number of words displayed. The results of this study suggest that

436 changing the number of words displayed may alter the perception of ease of text

437 input in persons with SCI but does not have an objective influence on TIS. Further

438 studies should be carried out to evaluate the influence of other WPS parameters

439 on TIS. These results are important for health-related professionals whose role is

440 to advise persons with SCI in the choice of word prediction software. It seems

441 important to reduce the number of words displayed for persons with low cervical

442 SCI, or not to use WPS at all, and to increase the number of words displayed for

443 persons with high cervical SCI in order to increase the comfort of use of WPS.

444 However, it must be kept in mind that these results are based on a single data

445 collection session. It would be useful to evaluate the impact of specific training on

446 the influence of WPS. The impact of other parameters of word prediction software

447 should also be considered in further studies, such as the location of the prediction 
448 list and the feature of only suggesting words of 5 characters or more, to decrease 449 visual search time.

450

451 
1. Folan A, Barclay L, Cooper C, Robinson M. Exploring the experience of clients with tetraplegia utilizing assistive technology for computer access. Disabil. Rehabil. Assist. Technol. [Internet]. 2015 [cited 2014 Jul 9];10:4652. Available from: http://www.ncbi.nlm.nih.gov/pubmed/24050283

2. Bigot P, Croutte E. La diffusion des technologies de 1' information et de la

6. Kim DG, Lee BS, Lim SE, Kim DA, Hwang S Il, Yim YL, et al. The communication dans la société. Rapp. réalisé à la demande du Cons. Général des Technol. l'Information (Ministère l'Economie, des Financ. l'Emploi) l'Autorité Régulation des Commun. Electron. des Postes. [Internet]. Paris : Centre de Recherche pour l'Etude et l'Observation des Conditions de Vie, 2014, 220 p. Available from : http://www.credoc.fr/pdf/Rapp/R317.pdf

3. Laffont I, Biard N, Bouteille J, Pouplin S, Guillon B, Bernuz B, et al. Tétraplégie: solutions technologiques de compensation des incapacités découlant de l'atteinte des membres supérieurs. La Lett. médecine Phys. réadaptation [Internet]. 2008 [cited 2012 Dec 6];24:113-21. Available from: http://www.springerlink.com/index/10.1007/s11659-008-0106-y

4. Betke M, Gips J, Fleming P. The camera mouse: visual tracking of body features to provide computer access for people with severe disabilities. IEEE Trans. Neural Syst. Rehabil. Eng. [Internet]. 2002;10:1-10. Available from: http://www.ncbi.nlm.nih.gov/pubmed/12173734

5. Huo X, Park H, Kim J, Ghovanloo M. A dual-mode human computer interface combining speech and tongue motion for people with severe disabilities. IEEE Trans. Neural Syst. Rehabil. Eng. [Internet]. 2013 [cited 2015 May 11];21:979-91. Available from: http://www.ncbi.nlm.nih.gov/pubmed/23475380 selection of the appropriate computer interface device for patients with high cervical cord injury. Ann. Rehabil. Med. 2013;37:443-8.

7. Kim J, Park H, Bruce J, Rowles D, Holbrook J, Nardone B, et al. Qualitative assessment of tongue drive system by people with high-level spinal cord injury. J. Rehabil. Res. Dev. [Internet]. 2014 [cited 2015 May 11];51:451-65. Available from: http://www.ncbi.nlm.nih.gov/pubmed/25019667 
8. Choi C, Na Y, Rim B, Kim Y, Kang S, Kim J. An SEMG computer interface using three myoelectric sites for proportional two-dimensional cursor motion control and clicking for individuals with spinal cord injuries. Med. Eng. Phys. [Internet]. 2013 [cited 2015 May 11];35:777-83. Available from: http://www.ncbi.nlm.nih.gov/pubmed/22939517

9. Turpin G, Armstrong J, Frost P, Fine B, Ward C, Pinnington L. Evaluation of alternative computer input devices used by people with disabilities. J. Med. Eng. Technol. [Internet]. 2005;29:119-29. Available from: http://www.ncbi.nlm.nih.gov/pubmed/16019881

10. Le Pévédic B. Prédiction Morphosyntaxique Évolutive HandiAS, Doctoral Thesis, Ecole Doctorale Sciences pour l'Ingénieur, 1997, Nantes, 142p.

11. Pouplin S, Robertson J, Antoine J, Blanchet A, Kahloun JL, Engineer D, et al. Effect of dynamic keyboard and word-prediction systems on text input speed in persons with functional tetraplegia. J. Rehabil. Res. Dev. 2014;51:467-80.

12. Raynal M, Vigouroux N. Genetic algorithm to generate optimized soft keyboard. CHI '05 Ext. Abstr. Hum. factors Comput. Syst. - CHI '05 [Internet]. 2005;1729. Available from: http://portal.acm.org/citation.cfm?doid=1056808.1057008

13. Kushler C. AAC using a reduced keyboard. Proc. CSUN Conf. Technol. Pers. with Disabil. CSUN'98. Calif. State Univ. Nortridge CA. 2001;

14. Isokoski P. Performance of menu-augmented soft keyboards. Proc. 2004 Conf. Hum. factors Comput. Syst. - CHI '04 [Internet]. 2004;423-30. Available from: http://portal.acm.org/citation.cfm?doid=985692.985746

15. Harbusch. An evaluation an evaluation study of two button scanning with ambiguous keyboards. In: 7th Conference for the Advancement of Assistive Technology in Europe, AATE'2003. Dublin, Ireland. 2003.

16. Rieger JM. The effect of automatic speech recognition systems on speaking workload and task efficiency. Disabil. Rehabil. [Internet]. 2003 [cited 2013 Jul 2];25:224-35. Available from: http://informahealthcare.com/doi/abs/10.1080/0963828021000030855

17. Pouplin S, Roche N, Hugeron C, Isabelle V, Bensmail D. Recommendations and settings of word prediction software by healthrelated professionals for people with spinal cord injury $\square$ : a prospective observational study. Eur. J. Phys. Rehabil. Med. 2015; article in press. 
18. Wandmacher T, Antoine J, Poirier F, Départe J-P. Sibylle, An Assistive Communication System Adapting to the Context and Its User. ACM Trans. Access. Comput. 2008;

19. Koester HH, Levine SP. Effect of a Word Prediction Feature on User Performance. Augment. Altern. Commun. [Internet]. 1996;12:155-68. Available from: http://informahealthcare.com/doi/abs/10.1080/07434619612331277608

20. Laffont I, Dumas C, Pozzi D, Ruquet M, Tissier AC, Lofaso F, et al. Home trials of a speech synthesizer in severe dysarthria: patterns of use, satisfaction and utility of word prediction. J. Rehabil. Med. [Internet]. 2007 [cited 2012 Nov 16];39:399-404. Available from: http://www.ncbi.nlm.nih.gov/pubmed/17549332

21. Vigouroux N, Vella F, Truillet P, Raynal M. Evaluation of AAC for text input by two groups of subjects $\square$ : able-bodied subjects and disabled Motor Subjects. In: 8th ERCIM UI4All, Vienne (Autriche). 2004.

22. Anson D, Moist P, Przywara M, Wells H, Saylor H, Maxime H. The effects of word completion and word prediction on typing rates using on-screen keyboards. Assist. Technol. [Internet]. 2006;18:146-54. Available from: http://www.ncbi.nlm.nih.gov/pubmed/17236473

23. Anson D. The effect of word prediction on typing speed. Am. J. Occup. Ther. 1993;47:1039-42.

24. Koester HH. Learning and performance in scanning systems with and without word prediction - report on a pilot study. In: Proceedings of RESNA 1992 -. 1992.

25. Koester HH. The effect of a user's search strategy on performance with word prediction. In: Conference proceeding of RESNA. 1997.

26. Venkatagiri HS. Effect of Window Size on Rate of Communication in a Lexical Prediction AAC System. AAC Augment. Altern. Commun. 1994;10:105-12.

27. Koester HH, Levine S. Keystroke-level models for user performance with word prediction. Augment. Altern. Commun. [Internet]. 1997;13:239-57. Available from: http://informahealthcare.com/doi/abs/10.1080/07434619712331278068 
554

561

562

563

564

565

566

567

568

569

570

571

572

573

\section{TABLES}

28. Guttmann L. Spinal Cord Injuries, Comprehensive Management and Research. 1ere éditi. Blackwell Science Ltd; 1973.

29. Fraval Lye M, Boutard C. Textzados. Ortho Edit. Isbergues: 2004.

30. Tam C, Wells D. Evaluating the benefits of displaying word prediction lists on a personal digital assistant at the keyboard level. Assist. Technol. [Internet]. 2009 [cited 2012 Dec 6];21:105-14. Available from: http://www.ncbi.nlm.nih.gov/pubmed/19908678

31. Wandmacher T, Antoine J, Poirier F, Départe J-P. Sibylle, An Assistive Communication System Adapting to the Context and Its User. ACM Trans. Access. Comput. 2008;1:1-30. 
575 Table 1. Characters per minute - Mean (sd)

576 Table 2. Number of errors - Mean (sd)

577 Table 3. Key selection speed (key presses per minute) - Mean (sd)

578 Table 4. Rate of word prediction use- Mean (sd)

579

580 